\title{
Efectos agudos de distintas estrategias de calentamiento sobre el rendimiento físico y las habilidades motrices en escolares Acute effects of different warm-up strategies on physical performance and motor skills in schoolchildren

\author{
*Nicolás Gómez-Álvarez, *Alexander Schweppe-Villa, *Aarón Parra-Gatica, *Falú Cid-Rojas, *Gustavo \\ Pavez-Adasme, *,**Felipe Hermosilla-Palma \\ *U niversidad Adventista de Chile (Chile), *,**U niversidad Autónoma de Chile (Chile)
}

\begin{abstract}
Resumen: El objetivo de esta investigación fue valorar los efectos de distintos tipos de calentamientos aplicados en escolares sobre la calidad de movimiento de las habilidades motrices y el rendimiento de sal to y carrera. La metodología utilizada fue a partir de un diseño estudio cruzado aleatorizado ( $n=27$ escolares), que incluyó tres condiciones experimental es para val orar el efecto agudo de distintos tipos de cal entamiento (tradicional, basado en juegos adaptados reducidos y FIFA $11+$ ) sobre el salto vertical, sprint en 20 metros y habilidades motoras de locomoción y control de objetos en niños y niñas. Se utilizó estadística descriptiva y comparativa a través deAN OVA de medidas repetidas y Friedman con sus pruebas post-hoc según corresponda. Los resultados muestran que todos los tipos de cal entamiento mostraron diferencias significativas en sprint, salto vertical y habilidades motoras, respecto al a la condición control sin calentamiento. El calentamiento basado en juegos adaptados reducidos presentó efectos superiores que las otras modalidades en las habilidades motoras de locomoción ( $p<$ .05). Las comparaciones entre calentamientos mostraron que la intervención basada en FIFA 11+ mostró ser más eficaz en reducir el tiempo de sprint en 20 metros y aumentar la altura del salto vertical $(p<.05)$. En conclusión, este estudio sugiere que, la selección de una estrategia adecuada basada en una diversidad de experiencias motrices como las ofrecidas por los protocolos de calentamiento FIFA11+ y de juegos adaptados reducidos podría ser clave para mejorar el rendimiento motor y la magnitud de los beneficios asociados al calentamiento.
\end{abstract}

Palabras claves: educación física, competencia motriz, aprendizaje motor, rendimiento motor, rendimiento físico.

\begin{abstract}
The objective of this research is to assess the effects of different types of warm-ups applied in schoolchildren on the quality of movement of motor skills and jumping and sprint performance. The methodology used was based on a randomized crossover study design ( $n=27$ schoolchildren), which included three experimental conditions to assess the acute effect of different types of warm-up (traditional, based on reduced adapted games and FIFA 11+) on the vertical jump, sprint in 20 meters and motor skills of locomotion and control of objects in children. Descriptive and comparative statistics were used through repeated measures AN OVA and Friedman with its post-hoc tests as appropriate. The results show that all types of warm-up showed significant differences in sprint, vertical jump and motor skills, compared to the control condition without warm-up. The warm-up based on reduced adapted games presented greater effects than the other modalities on the motor skills of locomotion $(p<.05)$. Comparisons between warm-ups showed that the intervention based on FIFA $11+$ was shown to bemore effective in reducing sprint time by 20 meters and increasing vertical jump height $(p<.05)$. In conclusion, this study suggests that the selection of an appropriate strategy based on a diversity of motor experiences such as those offered by the FIFA11 + warm-up protocols and reduced adapted games could be key to improving motor performance and the magnitude of the benefits associated with warm-up.
\end{abstract}

Keywords: physical education, motor competence, motor learning, motor performance, physical performance.

\section{Introducción}

La O rganización M undial de la Salud (OMS) recomienda que niños y adolescentes deben completar en promedio 60 minutos de actividad física moderada a vigorosa (Bull et al., 2020), sin embargo, solo un 19\% cumple estas directrices a nivel global (Guthold et al.,

Fecha recepción: 15-12-20. Fecha de aceptación: 03-03-21

Gustavo Pavez-Adasme

gustavopavez@unach.cl
2020), y en particular en Chile se han reportado datos similares sobre el cumplimiento de las recomendaciones de movimiento para niños (27.4\%) y adolescentes (18.9\%) (Aguilar-Fariaset al., 2018), lo que podría afectar las dimensiones sociales, afectivas, mentales y biológicas (World Health $O$ rganization, 2020). Dado el impacto de la inactividad física sobre la salud, existe una preocupación transversal por la comunidad científica y los distintos profesionales de la actividad física para mejorar el diseño de estrategias que pudiesen ayudar a que niños y adolescentes se adhieran a un estilo de vida 
activo (Stamatakis \& Bull, 2020). Recientes propuestas señalan que los diferentes especialistas que interactúan en la planificación de intervenciones, no solo deben considerar la cantidad de actividad física, si no que deben tener preocupación por la calidad de ellas (Davids et al. , 2016; Rudd et al. , 2020), de tal manera que se logre una al fabetización física, dotando de competencias físicas, afectivas y cognitivas que permitan a los niños y adolescentes adherirse a un estilo de vida activo (Cornish et al., 2020; Rudd et al., 2020).

En este contexto, la educación física desempeña un rol determinanteal contribuir con experienciasmotrices que favorecen el rendimiento motor durante toda la etapa escolar (García-Hermoso et al., 2020) y adicionalmente, intervenciones basadas en habilidades motoras fundamentales, puede aumentar los niveles de actividad física y disminuir la conducta sedentaria de niños (Engel et al., 2018). Lamentablemente las clases de educación física muestran una bajaadherencia y tiempo de compromiso motor tanto en niños como adolescentes (Hollis et al., 2017; Reyes et al., 2020), lo que podría estar asociado a una baja motivación o baja percepción de autoeficacia (Arribas-Galarraga et al., 2020; Fraile et al., 2019; García et al., 2020; Wing et al., 2016). Dado el contexto actual, en el que cada vez existen menos oportunidades de acceder a un ambiente amigable que contribuya al desarrollo de habilidades motrices (Davids et al., 2016), es necesario que los profesores de educación física, diseñen sesiones que contribuyan a generar respuestas afectivas que motiven la participación y la percepción de autoeficacia con el fin de hacer de sus sesiones más efectivas (Baños et al., 2020; Delextrat et al., 2020; Lakicevic et al., 2020).

El calentamiento como parte inicial de una sesión de ejercicio físico, es una estrategia habitualmente utilizada con el objetivo de generar efectos agudos en la optimización del desempeño motor (Fradkin et al., 2010; Kar \& Banerjee, 2013), caracterizadas por un conjunto de actividades organizadas en relación a los objetivos principales de la sesión (M cGowan et al., 2015). En niños y adolescentes ha mostrado tener efectos agudos sobre el rendimiento en actividades lineales como velocidad, salto, fuerza o ejercicios de larga duración (Gómez-Álvarez et al., 2020; lacono et al., 2019), así como también en el desempeño en actividades de características no lineales como deportes, compuestos de cambios de velocidad y de dirección (Chatzopoulos et al. , 2014) y habilidades específicas de deportes (Frikha et al., 2017). Adicionalmente existe evidencia que el calentamiento podría colaborar a aumentar el control motor y el aprendizaje motor (Ajemian et al., 2010; Vleugels et al., 2020).

En educación física escolar, la evidencia sobre los efectos de estrategias de cal entamiento aún es limitada, y al gunos estudios han mostrado efectos agudos sobre la competencia de movimiento funcional (Coker, 2018), fuerza explosiva (Garrigós et al., 2020) y flexibilidad (Díaz-Soler et al., 2015), así como también al ser implementado de forma sistemática podría ser eficaz para mejorar la competencia motora de niños (Chang et al. , 2020; Font-Lladó et al ., 2020) 0 disminuir el riesgo de lesiones (Emery et al., 2020). Sin embargo, debido a los amplios desafíos motores, cognitivos, y motivacionales implicados en la clase de educación física y a sus diferentes objetivos según grupos etarios, es necesario identificar como distintas estrategias de calentamiento podrían ser empleadas por los profesores de educación física para mejorar la respuesta de los estudiantes a las actividades motoras planificadas. En este sentido, el objetivo de esta investigación es valorar los efectos de distintos tipos de calentamientos aplicados en escolares sobre la calidad de movimiento de las habilidades motrices y el rendimiento de salto y carrera.

\section{Material y método}

Se diseñó un estudio cruzado aleatorizado, que incluyó cuatro condiciones experimentales para valorar el efecto agudo del calentamiento en el rendimiento motor en niños y niñas.

\section{Participantes}

Se utilizó una muestra por conveniencia compuesta por veinte y siete estudiantes entre 9 y 10 años (edad, $9.33 \pm 0.48$ años; talla, $1.37 \pm 0.06 \mathrm{~cm}$; masa corporal, $38.61 \pm 8.25 \mathrm{~kg}$ ) pertenecientes al mismo nivel educa tivo (región de Ñuble, Chile) quienes aceptaron participar del estudio y cumplieron con los criterios de inclusión y exclusión. Criterios de inclusión: (i) participación regular de las clases de educación física escolar; (iii) firmar el asentimiento informado por parte de los participantes (ii) consentimiento informado de los padres o tutores firmado. Los criterios de exclusión fueron: (i) lesión musculo-esquelética o enfermedad incompatible con la práctica de ejercicio físico (ii) incumplimiento de las normas establecidas para la ejecución del diseño experimental.

Todoslosniñosy niñas participaron de formaal eatoria de todas las sesiones: (i) condición control sin calenta miento (CC); (ii) cal entamiento tradicional (CT); (iii) 
ccalentamiento basado en juegos adaptados reducidos (CJAR); 0 (iv) cal entamiento basado FIFA 11+ (CF11+), para luego real izar las pruebas de rendimiento motor, a saber, habilidades motoras (HM) y rendimiento físico (salto vertical y sprint 20 metros).

Todos los niños y niñas firmaron un asentimiento informado, mientras que los padres o tutores, por su parte, firmaron un consentimiento informado, resguardando las consideraciones éticas de la declaración de Helsinki (Asociación M édica Mundial, 1964)

\section{Procedimientos}

Los investigadores principales se comunicaron con la administración del colegio para presentar los objetivos del estudio y solicitar la autorización e invitar a participar a sus estudiantes. Durante una reunión con los apoderados o tutores, se informó de los procedimientos experimentales y sus objetivos, extendiendo una invitación a participar del estudio, solicitando el consentimiento informado y el asentimiento de los niños y niñas.

Todas las mediciones e intervenciones se realizaron durante la jornada escolar en las instalaciones deportivas del establecimiento educativo. Dos semanas antes del inicio de las sesiones experimentales se realizaron distintas medidas antropométricas y cuestionarios demográficos (ver figura 1). Adicionalmente cada participante se familiarizó con los diferentes tipos de calentamiento y con las distintas pruebas de rendimiento motor (TGMD-2, salto vertical y sprint 20 metros).

Las cuatro sesiones (control sin calentamiento y las tres experimentales) se realizaron en días no consecutivos con al menos 48 horas de diferencia, en los que se le solicitó a cada estudiante no participar de actividad física intensa. Cada calentamiento se real izó en grupos de 6 a 8 niños en una condición al eatorizado y luego de un descanso de 5 minutos realizar la prueba de salto horizontal, velocidad yTGMD-2 (ver figura 1).

Condición control sin calentamiento (CC); En esta sesión los participantes se mantuvieron en reposo por $18 \mathrm{mi}$ nutos, recibiendo explicaciones de los beneficios del calentamiento sobre distintas habilidades motoras y físicas.

CalentamientoTradicional (CT); El CT estuvo organiza do por movilidad articular por 5 minutos y 13 minutos de trote a un ritmo autoseleccionado. Generalmente las tareas del calentamiento tradicional suelen incluir ejercicios continuos aeróbicos de baja intensidad como un trote suave, movilidad articular y ejercicios de flexibilidad (McGowan et al. , 2015).
Cal entamiento basado en JuegosAdaptadosR educidos (CJAR); El calentamiento basado en juegos reducidos, se realizó por 18 minutos y estuvo compuesto por distintas adaptaciones a juegos tradicionales en el que se alteraron distintas reglas como: las dimensiones del terreno, número de jugadores, disminución del tiempo de dura ción de la actividad, pero sin perder la lógica del juego original, de esta manera generar juegos del alta intensidad en modal idades de 3 vs 304 vs 4 (Carrasco Beltrán et al., 2015). Para esta modalidad los juegos utilizados fueron: (i) Q uitar la cola, en el que cada niño se coloca un pañuelo en el pantalón en la zona lumbar baja, como si fuese una cola. Al sonido del silbato deben quitar la mayor cantidad de colas, si se la quitan pueden continuar jugando hasta el silbato de descanso; (ii) quitar la colaen columna, en el que losniños posicionados en una columna, uno tras otro tomados de los hombros, el primero de la fila frente a la columna quita el pañuelo al último de la fila, pueden rotar a indicación del investigador.

FIFA 11+ Kids (CF11+); estuvo basado en el protocolo de calentamiento creado por un grupo de expertos internacionales enfocado en la prevención de lesiones paraniños de 7 a 13 años que practican fútbol en Fédération Internationale de Football Association (FIFA 11+ for Kids Manual a Warm-up Programme for Preventing Injuries in Children's Football, 2016). De hecho esta modalidad ha mostrado efectos favorables para mejorar habilidades motoras como la agilidad, la dinámica de equilibrio, poder de salto y habilidades técnicas (Rössler et al., 2016). En esta modalidad fue implementada en $18 \mathrm{mi}-$ nutos y se seleccionaron ejercicios específicos de acuerdo a las recomendaciones del programa FIFA 11+ for Kids.

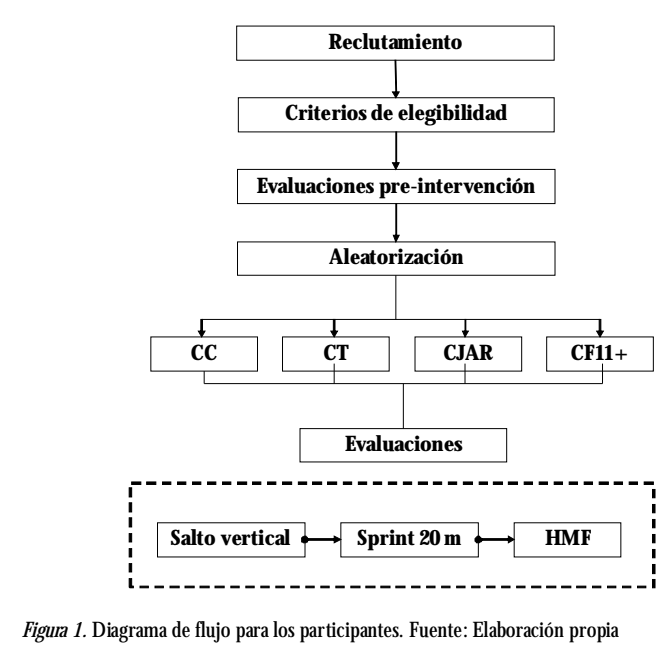

Instrumentos

El efecto agudo sobre el rendimiento motor fue rea 
lizado 5 minutos después del término del calentamiento manteniendo siempre el mismo orden en las evalua ciones: Salto vertical, sprint 20 metros y HMF.

Salto Vertical; fue evaluado mediante un sal to contramovimiento (CMJ), en el que cada participante ubicó sus manos en la cadera y los pies separados para luego realizar un salto vertical de máximo esfuerzo. Todos los niños y niñas fueron instruidos para flexionar las rodillas una vez realizara contacto con el suelo y a realizar un total de 3 intentos, considerando el mejor de ellos como para los análisis. La evaluación se realizó con una plataforma de contacto Chronojump (Chronojump Boscosystem, Barcelona, Spain) (Pueo et al. , 2020).

Sprint 20 metros; Dos fotocélulas (Chronojump Boscosystem, Barcelona, Spain) fueron ubicadas a 20 metros de distancia para medir el tiempo empleado en recorrer esa distancia. Cada niño, inició la carrera a 3 metros de la primera cámara, y a la indicación del evaluador corrió a su máximo esfuerzo. Cada participante completó un total de 3 intentos considerando el mejor para los análisis.

H abilidades motoras fundamentales; se utilizó la segunda versión del Test Gross M otor Development (TGMD-2), el cual está compuesto por dos sub-escal as; (i) habilidades locomotoras: correr, galopar, salto en un pie, saltar un obstáculo, salto horizontal y desplazamiento (galope) lateral; y (ii) habilidades de control de objetos: ba tear una bolaestática, dribleestático recepciones (UIrich $\&$ Sanford, 2000).

Es un test basado en criterios que evalúa cualitativamente el desempeño de las habilidades motoras gruesas, ha sido validado para población chilena (Cano-Cappellacci et al., 2015) y utilizado frecuentemente en Chile (Pavez-Adasme et al., 2020). El proceso de evaluación de cada habilidad se realizó mediante la grabación de video y posterior puntuación, procedimiento ampliamente utilizado y que ha mostrado una alta fiabilidad inter-evaluador y test-retest (Rey et al., 2020).

\section{Análisis estadístico}

Se utilizó estadísticos descriptivos utilizando media y deviación estándar (DE), adicionalmente se presenta la mediana para pruebas no paramétricas. Los supuestos de normal idad, homogeneidad de varianza y esfericidad fueron evaluados previamente para realizar estadísticas paramétricaso no paramétricas según corresponda. Para comparar las diferencias en relación a condición control sin calentamiento se utilizó ANOVA de medidas repe- tidas para sprint, CMJ y TGMD-2 mientras que se utilizó estadísticano paramétricadeFriedman paraTGMD$2 \mathrm{~L}$ y TGMD-2 CO. Las diferencias de medias en relación a CC fueron utilizadas para identificar diferencias entre las estrategias de calentamiento implementadas utilizando AN OVA de medidas repetidas para CMJ y pruebano paramétrica de Friedman parasprint, TGMD2, TGMD-2 L y TGMD-2 C0. Pruebas post-hoc de Bonferroni y Durbin-Conover fueron aplicados según correspondiera. Además, el tamaño del efecto fue calculado utilizando Hedges'g, interpretado cualitativamente como: $<0.2=$ trivial; $0.2-0.49=$ pequeño; $0.50-0.79=$ moderados; $y>0.8=$ grande (Fritz et al., 2012)

Todos los análisis estadísticos se realizaron en Jamovi ®, versión 1.2 (The jamovi project, 2021), el nivel de significancia se estableció para un valor $p<.05$.

\section{Resultados}

En total 27 estudiantes participaron de este estudio (edad, $9.33 \pm 0.48$ años; talla, $1.37 \pm 0.06 \mathrm{~cm}$; masa corporal, $38.61 \pm 8.25 \mathrm{~kg})$. Ver tabla 1 .

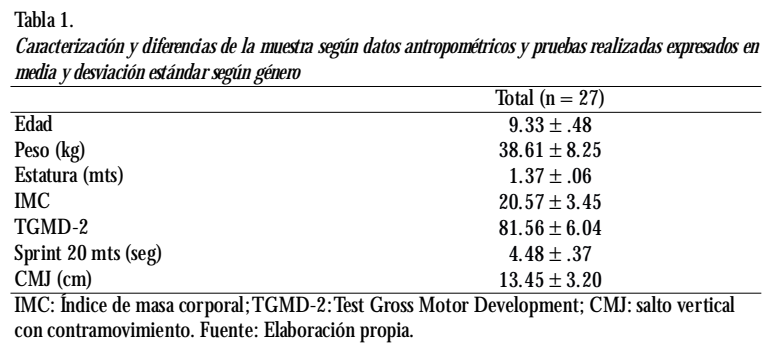

\section{Diferencias en relación a condición control sin calentamiento.}

La tabla 2, muestra los resultados de la CC y las tres estrategias de calentamiento utilizadas CT, CF11+ y CJAR. El análisis estadístico de medidas repetidas mostró diferencias significativas entre los puntajes totales para sprint $\left(F(3,78)=8.03, p<0.001, X^{2}=0.24\right), C M J$ $\left(F(3,78)=9.12, p<0.001, X^{2}=0.26\right), T G M D-2$ $\left(F(3,78) 11.9, p<0.001, X^{2}=0.31\right), T G M D-2 L\left(X^{2}(3)\right.$ $=17,3 ; p<0.001)$ y TGMD $-2 \mathrm{CO}\left(X^{2}(3)=19,2\right.$; $p<0.001)$.

Los resultados de la prueba post-hoc de Bonferroni para sprint no revelaron diferencias significativas con $C C(p>0.05)$, con tamaños de efecto moderados para CT y CF11+ y pequeños en CJAR, adicionalmente se encontraron diferencias significativas entre los otros calentamientos (CT vsCF11+ [DM =0.25, $p<0.01$ ]; CF11+ vs C]AR [DM = -0.14, $p=0.046])$. Para CM] la prueba post-hoc reveló diferencias significativas en- 
tre CC vs CT $(p=0.04)$ y CF11+ $(p<0.001)$, con tamaños de efecto de moderados (CT y CJAR) a grandes (CF11+), además se encontraron diferencias entre AF11+ y CJAR ( $p=0.046)$.

Los resultados sobre habilidades motoras fundamentales mostraron diferencias significativas en el puntaje global deTGMD-2 para todas las estrategias de calenta miento en relación a CC (CT: $D M=2.56, p=0.002$; CF11+: DM =3.41, $p<0.001 ;$ CJAR: DM = 3.63, $p<$ 0.001) y tamaños de efecto de pequeño (CT) a modera dos (CF11+ y CJAR). Para las sub-pruebas deTGMD2 L y TGMD-2 CO la prueba post-hoc de DurbinConover mostró diferencias significativas entre CC con todas las estrategias de calentamiento (TGM D-2 L [CT: $D M=0.85$, Mediana: 42 vs 42, $p=0.047 ;$ CF11+: DM $=1.00$, M ediana: 42 vs42, $p=0.02 ;$ CJAR: $D M=1.67$, Mediana: 42 vs 44, $p<0.001$ ]; TGMD-2 CO [CT: DM $=1.70$, Mediana: 40 vs 42, $p=0.006$; CF11+: $D M=$ 2.41, M ediana: 40 vs 43, $p<0.001 ;$ CJAR: $D M=1.96$, Mediana: 40 vs 42, $p=0.003]$ ).
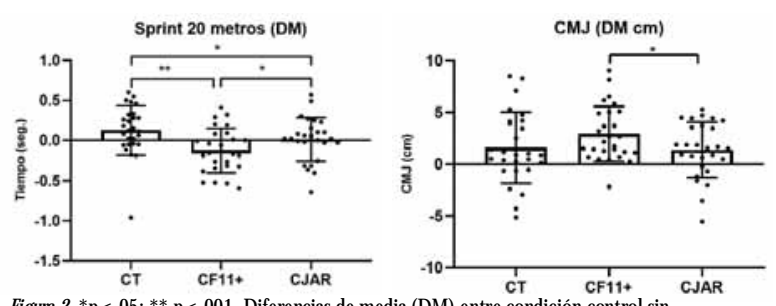

Figura $2 .{ }^{*} p<.05 ; * * p<.001$. Diferencias de media (DM) entre condición control sin calentamiento y estrategias de calentamiento para sprint en 20 metro y CMJ. Fuente: Elaboración propia.

Los resultados en habilidades motoras solo mostra ron diferencias la sub-prueba de locomoción entre CT (media $=.85 \pm 2.51$; mediana $=.00$ ) y CJAR (media=1.67 \pm 1.98; mediana $=2.00$ ), valor $p=.01$ y un TE moderado (.36 [IC 95\%.03, .71]) y entre CJAR y CF11+ (media $=1.00 \pm 2.15$; mediana $=.00$ ), valor $p$ $=.03$ y unTE moderado ( .32 [IC 95\% - .01, .67]). Ver figura 3.

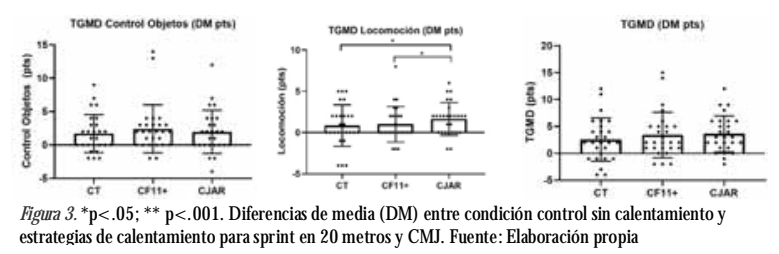

Tabla 2

Resul tados en rendimiento físico y competencia motora en condición control sin calentamiento en comparación a los distintos tipos de calentamiento \begin{tabular}{ccccccc}
\hline CC & CT & CC vSCT & CF11t & CC vsCF11t & CJAR & CC vsCJAR \\
Media DE & Media DE & ES (IC) & Media DE & ES (IC) & Media D DE & ES (IC)
\end{tabular} \begin{tabular}{llllll}
\hline Rendimiento físico & $13.45+3.20$ & $15.04+3.17$ & $.49(.08, .94)^{*}$ & $16.38+3.57 .85(.54,1.23)^{* *} 14.85+3.32$ & $.42(.11, .77)$
\end{tabular} $\begin{array}{lllllllll}\mathrm{CM})(\mathrm{cm}) & 13.45 \pm 3.20 & 15.04 \pm 3.17 & .49(.08, .94)^{*} & 16.38 \pm 3.57 & .85(.54,1.23)^{* *} & 14.85 \pm 3.32 & .42(.11, .77) \\ \text { Sprint (seg) } & 4.48 \pm 0.37 & 4.60 \pm 0.39 & 0.33(.02, .66) & 4.35 \pm .31 & -.37(-.70,-.07) & 4.49 \pm 0.37 & .04(-.24, .32)\end{array}$ Sprint (seg) TGMD-2 $81.56 \pm 6.0484 .11 \pm 4.18 .48(.22, .80) * 84.96 \pm 3.06 .70(.43,1.03) * 85.19 \pm 4.34 .68(.45, .97)$ $\begin{aligned} & { }^{*} \mathrm{p}<0.05 ; * * \\ & 1\end{aligned} \quad 40.15 \pm 4.5641 .85 \pm 3.13 .43(.18, .71)^{*} 42.56 \pm 2.10 .67(.34,1.05)^{* *} 42.11 \pm 2.64 .52(.23, .85)^{*}$ 11+; CJAR: calentamiento juegos adaptados reducidos; CMJ: salto vertical con contramovimiento; TGM D-2: Test Gross Motor Development puntaje total; TGMD-2 L: Test Gross Motor Development Locomoción; TGMD-2 C0: Test Gross Motor Development Control de objetos. Fuente: Elaboración propia.

\section{Comparaciones entre estrategias de calenta- miento.}

La figura 1 muestra las diferencias de medias en relación a CC utilizadas para comparar la efectividad de las estrategias de calentamiento, los resultados mostra ron diferencias significativas en sprint $\left(X^{2}(2)=20.2 ; p\right.$ $\left.<.001), C M J(F(2.52))=4.58, p<.015, X^{2}=.15\right)$ y TGMD-2L $\left(X^{2}(2)=6.86 ; p=0.03\right)$. En sprint CT (me$\mathrm{dia}=.13 \pm .31$; mediana $=.12$ ) mostró diferencias significativas con CF11 + (media $=-.12 \pm .27$; mediana $=$ - .16) con un valor $p<0,001$ y unTE grande $(0,85$ [IC $95 \% ;-1.99,-.47]$ ) y con CJAR (media $=.01 \pm .27$; mediana $=.02$ ) con un valor $p=.01$ y unTE moderado $(-0,38[$ [C $95 \%-0,72,-0,06])$, además CF $11+$ mostró diferencias significativas en relación a CJAR $(p=.00$; $\mathrm{TE}=.51[\mathrm{IC} 95 \%, 22, .85])$. Para CM] los resultados mostraron diferencias significativas para las diferencias entre CF11+ (media $=2.93 \pm 2.67$ ) y CJAR (media $=1.39 \pm 2.68)$ con un valor $p$ de .02 y un tamaño del efecto moderado (.57 [IC 95\% - .88, - .30]).

\section{Discusión}

El objetivo de estudio fue valorar los efectos de distintos tipos de calentamientos aplicados en escolares sobre la calidad de movimiento de las habilidades motrices y el rendimiento de salto y carrera. Los resultados encontrados muestran que laestrategiade calentamiento FIFA11+ es efectiva para mejorar el desempeño en pruebas de velocidad y salto, al mismo tiempo que la intervención basa da en juegos adaptados reducidos (CJAR) mejora la ca lidad de ejecución de las habilidades motrices, principalmente locomoción

Los efectos agudos del calentamiento sobre el rendimiento físico han sido descritos previamente en diversas poblaciones (Fradkin et al., 2010; Kar \& Banerjee, 2013; M cGowan et al., 2015). Los resultados de nuestra investigación muestran diferencias estadísticamente significativas para CMJ no así para sprint, con tamaños del efecto grandes y pequeños, respectivamente. Sin embargo, es preciso especificar algunos puntos en particular. Los efectos reportados en sprint difieren de lo reportado por Rössler et al. (2016), quienes llevaron a cabo una intervención basada en el programa FIFA 11+ Kids, en futbolistas infantiles (edad $10.0 \pm 1.8$ años, frecuencia de entrenamiento tres sesiones semanales), estos fueron eval uados sobre diferentes variables de índo- 
le técnico y de rendimiento físico, siendo el sprint en 20 metrosaquellaque arrojó el menor tamaño del efecto (- .1), no observándose diferencias estadísticamente significativas respecto del grupo control sin calentamiento, lo que podría ser explicado debido al estatus deportivo de los sujetos deestudio. En este sentido, Wernbom, Augustsson \& Thome (2007), describen que los niveles de adaptación al ejercicio disminuyen a medida que se incrementa la condición deportiva del individuo aspecto que podría explicar los menores efectos reportados. Respecto de la altura de salto, se observaron mejoras significativas en esta variable a partir de los protocolos CT y CF11+, reportándose un tamaño del efecto grande $(T E=.85)$ en este último. El protocolo basado en juegos adaptados reducidos (CJAR) generó cambios pequeños (TE = .42) y estadísticamente significativos $(p<.05)$, respecto del grupo control sin cal entamiento. Estos resultados concuerdan con los hallazgos reporta dos en la literatura, en donde estrategias basadas en juegos reducidos, generaron incrementos en esta manifestación, por sobre otras modalidades de calentamiento (Zois et al., 2011). En la misma línea, Gelen (2011), comparó los efectos agudos de dos protocolos en niños (edad 13.3 \pm .5 años), participantes en escuelas deportivas, sobre la altura de salto evaluada mediante CMJ, en una de ellas se realizaron estiramientos estáticos y la otra se basó en ejercicios dinámicos; el rendimiento en el salto fue mejorado en ambos grupos, siendo estas diferencias estadísticamente significativas. Estos hallazgos se condicen con los propios, debido a que el grupo CT y CF11+ obtuvieron las mismas ganancias de rendimiento al comparar con el grupo CC. Como contra parte, Coledam et al. (2012), no reportaron diferencias estadísticamente significativas en CMJ entre una intervención basada en juegos y otra realizada a partir de ejercicios dinámicos, en esta investigación no hubo contraste con CC, por tanto, no es posible realizar una comparación de la magnitud del efecto, sin embargo, los autores concluyen sobre los beneficios de ambosprotocolos, sugiriendo que ambas modalidades podrían ser adecuadas para la mejora aguda del rendimiento físico.

Los resultados del TGMD sugieren la importancia del calentamiento en la ejecución de distintas habilida des motoras tanto de locomoción como de control de objetos, tales resultados invitan a ampliar los beneficios del calentamiento y su rol en el aprendizaje de nuevas habilidades motoras. El calentamiento no solo prepara músculos y tendones para el estrés motor, sino que, predispone al cuerpo hacia una habilidad motriz eficiente, favoreciendo el aprendizaje motor y reduciendo el error 0 «ruido» de la tarea o acción motora. Los resultados muestran mejoras significativas $(p<.05)$ en los tres tipos de calentamientos (CT, CF11+ y CJAR) respecto al control sin calentamiento (CC) para el puntaje total y la habilidad de control de objetos, mientras que el comportamiento se repite de igual forma para el apartado de locomoción, pero con una significancia mayor entre CC y CJAR ( $p<.001)$, esta última diferencia puede ser explicada por Ajemian et al. (2010), debido a la similitud entre la evaluación (TGMD) y el patrón de comportamiento de la habilidad motora expresada en CJAR, mientras la experiencia o gesto motor sea más cercano ala modalidad de evaluación (TGMD), el aprendizaje motor será evidenciado en la eficiencia de la acción motriz.

Alibazi et al. (2021) sugiere que el ejercicio agudo como de fuerza o resistencia aeróbica podrían favorecer una excitabilidad intra-cortical para modular una respuesta motora más efectiva, adicionalmente, incorporar diversas experiencias motrices de carácter no lineales, como ocurre con el CF11+ y CJAR, favorecería el aprendizaje motor y la calidad de movimiento de la habilidad motriz (Ajemian et al., 2010; Vleugels et al., 2020), además, como concluye Engels y Freund (2020), una mejor competencia percibida puede mediar el aumento del disfrute, que a su vez, fomenta la alfabetización física de los escolares.

Es preciso mencionar al gunas limitaciones presentes en este estudio: (i) no haber controlado a intensidad del ejercicio, aspecto que impide establecer la influencia de esta variable con mayor precisión sobre las repuestas agudas investigadas; (ii) el análisis del efecto de la maduración biológica sobre la efectividad del calentamiento; (iii) los efectos de la dinámica escolar al ser un estudio en contexto ecológico.

\section{Conclusión}

Según los resultados de este estudio la implementación del calentamiento puede ser efectiva para mejorar de manera transitoria la calidad de movimiento de las habilidades motrices y el rendimiento en salto vertical y carrera de 20 metros. Así mismo, la selección de una estrategia adecuada basada en una diversidad de experiencias motrices como las ofrecidas por los protocolos CF11t y CJAR podría ser clave para mejorar el rendimiento motor y la magnitud de los beneficios asociados al calentamiento.

Futuras investigaciones deberían valorar más estra- 
tegias que permitan generar un efecto agudo positivo, aportando evidencia sobre una diversidad metodológica aplicable al calentamiento en un contexto de Educación Física Escolar.

\section{Agradecimientos}

El equipo de investigadores agradece la participa ción y voluntad tanto de la comunidad estudiantil como de la administración del Colegio Hispano Americano de Chillán por su apertura y aporte al desarrollo de este estudio.

\section{Conflictos de interés}

Los investigadores declaran no tener conflictos de interés.

\section{Referencias}

Aguilar-Farias, N., Martino-Fuenteal ba, P. , CarcamoO yarzun, J., Cortinez-O 'Ryan, A., Cristi-M ontero, C. , Von 0 etinger, A., \& Sadarangani, K. P. (2018). A regional vision of physical activity, sedentary behaviour and physical education in adolescents from Latin America and the Caribbean: results from 26 countries. International Journal of Epidemiology, 47(3), 976- 986. https:/ / doi.org/ 10.1093/ ije/ dyy033

Ajemian, R., D'Ausilio, A., M oorman, H., \& Bizzi, E. (2010). W hy professional athletes need a prolonged period of warm-up and other peculiarities of human motor learning. Journal of M otor Behavior, 42(6), 381$388 . \quad$ https: / / doi.org/ 10.1080/ 00222895.2010 .528262

Alibazi, R., Pearce, A., Rostami, M., Frazer, A., Brownstein, C., \& Kidgell, D. (2021). Determining the Intracortical Responses After a Single Session of Aerobic Exercise in Young Healthy Individuals. Journal of Strength and Conditioning Research, 35(2), 562-575. https:// doi.org/ 10.1519/ jsc. 0000000000003884

Arribas-Galarraga, S., Luis-de Cos, I., Luis-de Cos, G., \& Urrutia-Gutierrez, S. (2020). Mediation Effect of Perceived Fitness on the Relationship between SelfEfficacy and Sport Practice in Spanish Adolescents. International Journal of Environmental Research and Public Health, 17 (23), 8800. https:// doi.org/ 10.3390/ ijerph17238800

Asociación Médica Mundial. (1964). Declaración de H elsinki de la AM M - Principios éticos para las investigaciones médicas en seres humanos.

Baños, R., Fuentesal, J., Conte, L., O rtiz-Camacho, M. del M., \& Zamarripa, J. (2020). Satisfaction, Enjoyment and Boredom with Physical Education as Mediator between Autonomy Support and Academic Performance in Physical Education. International Journal of Environmental Research and Public H ealth, 17(23), 8898. https: / / doi.org/ 10.3390/ ijerph17238898

Bull, F. C., Al-Ansari, S. S., Biddle, S., Borodulin, K., Buman, M. P., Cardon, G., Carty, C., Chaput, J. P., Chastin, S., Chou, R., Dempsey, P. C., DiPietro, L., Ekelund, U., Firth, J., Friedenreich, C. M., Garcia, L., Gichu, M., Jago, R., Katzmarzyk, P. T., ... W illumsen, J. F. (2020). World H ealth 0 rganization 2020 guidelines on physical activity and sedentary behaviour. British Journal of Sports M edicine, 54 (24), 1451-1462. https:/ / doi.org/ 10.1136/ bjsports-2020-102955

Cano-Cappellacci, M., Leyton, F. A., \& Carreño, J. D. (2015). Content validity and reliability of test of gross motor development in Chilean children. Revista de Saude Publica, 49(97). https:/ / doi.org/ 10.1590/ S00348910.2015049005724

Carrasco Beltrán, H., Reigal Garrido, R. E., Ulloa Díaz, D., Jesús Chirosa Ríos, I., \& Chirosa Ríos, L. J. (2015). Efecto de los juegos reducidos en la composición corporal y la condición física æeróbica en un grupo deadolescentes escolares. Revista M edica deChile, 143(6), 744-750. https:// doi.org/ 10.4067/ S003498872015000600007

Chang, N.-J., Tsai, I.-H., Lee, C.-L., \& Liang, C.H. (2020). Effect of a Six-Week Core Conditioning as a Warm-Up Exercise in Physical Education Classes on Physical Fitness, Movement Capability, and Balance in School-Aged Children. International Journal of Environmental Research and Public Health, 17(15), 5517. https: / / doi.org/ 10.3390/ ijerph17155517

Chatzopoulos, D., Galazoulas, C., Patikas, D., \& Kotzamanidis, C. (2014). Acute effects of static and dynamic stretching on balance, agility, reaction time and movement time. Journal of Sports Science and M edicine, 13(2), 403-409. http:/ / www.jssm. org

Coker, C. A. (2018). Improving Functional Movement Proficiency in Middle School Physical Education. Research Quarterly for Exercise and Sport, 89(3), 367- 372.

\subsubsection{6}

https: / / doi.org/ 10.1080/

Coledam, D. H. C., Paludo, A. C., De Oliveira, A. R., \& Dos-Santos, J. W. (2012). Dynamic exercise versus tag game warm up: The acute effect on agility and vertical jump in children. Journal of H uman Sport and 
Exercise, 7(1), 243-253. https:// doi.org/ 10.4100/ jhse. 2012.71.03

Cornish, K., Fox, G., Fyfe, T., Koopmans, E., Pousette, A., \& Pelletier, C. A. (2020). Understanding physical literacy in the context of health: a rapid scoping review. BMC Public H ealth, 20(1), 1569. https: / / doi. org/ 10.1186/s12889-020-09583-8

Davids, K., Araújo, D., \& Brymer, E. (2016). Designing Affordances for Health-Enhancing Physical Activity and Exercise in Sedentary Individuals. Sports Medicine, 46(7), 933-938. https:/ / doi.org/ 10.1007/ s40279-016-0511-3

Delextrat, A., Esser, P., Beale, N., Bozon, F., Eldridge, E., Izadi, H., Johansen-Berg, H., W heatley, C. , \& Dawes, H. (2020). Effects of gender, activity type, class location and class composition on physical activity levels experienced during physical education classes in British secondary schools: a pilot cross-sectional study. BMC Public H ealth, 20(1590). https:/ / doi.org/ 10.1186/ s12889-020-09698-y

Díaz-Soler, M., Vaquero-Cristóbal, R., EspejoAntúnez, L. , \& López-Miñarro, P. (2015). The effect of a warm-up protocol on the sit-and-reach test score in adolescent students. N utr H osp, 31 (6), 2618-2623. https:/ / doi.org/ 10.3305/ nh.2015.31.6.8858

Emery, C. A., Van Den Berg, C., Richmond, S. A., Palacios-Derflingher, L., M cKay, C. D., Doyle-Baker, P. K., McKinlay, M., Toomey, C. M., Nettel-Aguirre, A., Verhagen, E., Belton, K., MacPherson, A., \& Hagel, B. E. (2020). Implementing a junior high school-based programme to reduce sports injuries through neuromuscular training (iSPRINT): A cluster randomised controlled trial (RCT). British Journal of Sports M edicine, 54(15), 913-919. https:/ / doi. org/ 10.1136/ bjsports2019-101117

Engel, A. C., Broderick, C. R., van Doorn, N., Hardy, L. L., \& Parmenter, B. J. (2018). Exploring the Relationship Between Fundamental Motor Skill Interventions and Physical Activity Levels in Children: A Systematic Review and Meta-analysis. Sports M edicine, 48(8), 1845-1857. https: / / doi.org/ 10.1007/ s40279018-0923-3

Engels, E. S., \& Freund, P. A. (2020). Effects of cooperative games on enjoyment in physical education- How to increase positive experiences in students? PLOS ONE, 15(12), e0243608. https:/ / doi. org/ 10.1371/ journal. pone. 0243608

FIFA $11+$ for kids manual a warm-up programme for preventing injuries in children's football. (2016). https:/ / www.fifamedicalnetwork.com/ wp-content/ uploads/ cdn/ fifallplus_kids_booklet.pdf

Font-Lladó, R., López-Ros, V., M ontalvo, A. M., Sinclair, G., Prats-Puig, A., \& Fort-Vanmeerhaeghe, A. (2020). A Pedagogical A pproach to Integrative NeuromuscularTraining to Improve M otor Competence in Children: A Randomized ControlledTrail. Journal of Strength and Conditioning Research, 34(11), 3078-3085. https:/ / doi.org/ 10.1519/ JSC.0000000000003772

Fradkin, A. J. , Zazryn, T. R. , \& Smoliga, J. M . (2010). Effects of warming-up on physical performance: $A$ systematic review with meta-analysis. Journal of Strength and Conditioning Research, 24(1), 140-148. https:/ / doi. org/ 10.1519/ JSC.0b013e3181c643a0

Fraile, J., Tejero-González, C., Esteban-Cornejo, I., \&Veiga, Ó . (2019). Asociación entredisfrute, autoeficacia motriz, actividad física y rendimiento académico en educación física (Association between enjoyment, motor self-efficacy, physical activity and academic performance in physical education). Retos, 36(36), 58-63. https:/ / doi.org/ 10.47197/ retos. v36i36.63035

Frikha, M., Derbel, M. S., Chaâri, N., Gharbi, A., $\&$ Chamari, K. (2017). Acute effect of stretching modalities on global coordination and kicking accuracy in 12-13 year-old soccer players. Human M ovement Science, 54, 63-72. https:// doi.org/ 10.1016/ j.humov. 2017.03.008

Fritz, C. O., Morris, P. E., \& Richler, J. J. (2012). Effect size estimates: Current use, calculations, and interpretation. Journal of Experimental Psychology: General, 141(1), 2-18. https:/ / doi.org/ 10.1037/ a0024338

García-Hermoso, A., Alonso-M artínez, A. M., Ramírez-Vélez, R., Pérez-Sousa, M. Á., Ramírez-Campillo, R., \& Izquierdo, M. (2020). Association of Physical Education with Improvement of H eal th-R elated Physical Fitness 0 utcomes and Fundamental M otor Skillsamong Youths: A Systematic Review and Meta-analysis. JAM A Pediatrics, 174(6), e200223- e200223. https:/ / doi. org/ 10.1001/ jamapediatrics.2020.0223

García, F., Herazo, Y., Sánchez, L., Barbosa, E., Coronado, A., Corro, E., Villarreal, A., \& Redondo, C. (2020). Autoeficacia hacia la actividad física en escolares colombianos (Self-efficacy towards physical activity in Colombian schoolchildren). Retos, 38(38), 390-395. https:/ / doi.org/ 10.47197/ retos. v38i38.73878

Garrigós, Durán, Á. \& Sánchez-Pay, A. (2020). Influencia de dos tipos de calentamiento sobre la fuerza explosiva del tren inferior en estudiantes de Educación Física. Pensar En M ovimiento: Revista de Ciencias Del Ejercicio y La Salud, 18(1), e34705. https:/ / doi.org/ 10.15517/ pensarmov.v18i1.34705 
Gelen, E. (2011). Acute effects of different warm-up methods on jump performance in children. Biology of Sport, 28(2), 133-138. https:/ / doi.org/ 10.5604/ 947456

Gómez-Álvarez, N., M oyano, F., Huichaqueo, E., Veruggio, M., Urrutia, V., HermosillaPalma, F., \& PavezAdasme, G. (2020). Effects of theinclusion of jump exercises with and without external overload in the warm-up on physical performance parametersin young handball athletes. MHSalud, 17(1). https:/ / doi.org/ 10.15359/ mhs.17-1.4

Guthold, R., Stevens, G. A., Riley, L. M., \& Bull, F. C. (2020). Global trends in insufficient physical activity among adolescents: apooled analysis of 298 population-based surveys with 1.6 million participants. The Lancet Child and Adolescent Health, 4(1), 23-35. https:/ / doi.org/ 10.1016/ S23524642(19)30323-2

Hollis, J. L., Sutherland, R., W illiams, A. J., Campbell, E., Nathan, N., Wolfenden, L., M organ, P. J., Lubans, D. R., Gillham, K., \& W iggers, J. (2017). A systematic review and meta-analysis of moderate-to-vigorous physical activity levels in secondary school physical education lessons. International Journal of Behavioral Nutrition and Physical Activity, 14(1), 52. https:/ / doi.org/ 10.1186/ s12966-017-0504-0

Iacono, A., Vigotsky, A., Laver, L., \& Halperin, I. (2019). Beneficial Effects of Small-Sided Games as a Conclusive Part ofWarm-up RoutinesinYoung EliteH andball Players. Journal of Strength and Conditioning Research.

Kar, S., \& Banerjee, A. (2013). Influence of Active \& PassiveWarmingU $p 0 \mathrm{n}$ M otor Performance of theAthletes. IOSR Journal of Humanities And Social Science (IOSR-JHSS, 10(6), 21. www.losrjournals.0 rg

Lakicevic, N., Gentile, A., Mehrabi, S., Cassar, S., Parker, K., Roklicer, R., Bianco, A., \& Drid, P. (2020). Make Fitness Fun: Could Novelty Be the Key Determinant for Physical Activity Adherence? Frontiers in Psychology, 11, 577522. https:/ / doi.org/ 10.3389/ fpsyg 2020.577522

McGowan, C. J., Pyne, D. B., Thompson, K. G., \& Rattray, B. (2015). Warm-Up Strategies for Sport and Exercise: Mechanisms and Applications. Sports M edicine, 45(11), 1523-1546. https:/ / doi.org/ 10.1007/ s40279-0150376-x

Pavez-Adasme, G., Hernández-M osqueira, C., Torres, $S$., Paillaca, M., Concha, M., Cabrera, M., Concha, M., \& Gómez-Álvarez, N. (2020). Test de desarrollo motor aplicados en Chile entre el período 2014-2018. Unarevisión sistemática| Ciencias de laActividad FísicaU CM. Revista Ciencias de La Actividad Física U CM , 21(1), 1-11. http:/ / revistacaf.ucm.cl/ article/ view/ 366

Pueo, B. , Penichet-Tomas, A., \& Jimenez-O Imedo, J. (2020). Reliability and validity of the Chronojump open-source jump mat system. Biology of Sport, 37(3), 255-259. https:/
/ doi.org/ 10.5114/ biolsport.2020.95636

Rey, E., Carballo-Fazanes, A., Varela-Casa, C. \& \& AbelairasGómez, C. (2020). Reliability of the test of grossmotor development: A systematic review. PLOS ONE, 15(7 July). https:/ / doi.org/ 10.1371/ journal. pone.0236070 Reyes, A., Rivas, J., \& Pavez-Adasme, G. (2020). Tiempo de compromiso motor en la clase de educación física. Voces De La Educación, 5(10), 90-113. https:/ / www. revista. vocesdelaeducacion. com. mx/ index. php/ voces/ article/ view/ 256/ 213

Rösster, R., Donath, L., Bizzini, M., \& Faude, O. (2016). A new injury prevention programmefor children'sfootball - FIFA 11+ Kids - can improve motor performance: a cluster-randomised controlled trial. Journal of Sports Sciences, 34(6), 549-556. https:/ / doi.org/ 10.1080/ 02640414.2015 .1099715

Rudd, J. R., Pesce, C., Strafford, B.W., \& Davids, K. (2020). Physica Literacy - A Journey of Individual Enrichment: An Ecological Dynamics Rationale for Enhancing Performanceand PhysicalActivityinAll. Frontiersin Psychology, 11(1904). https:/ / doi.org/ 10.3389/ fpsyg, 2020.01904

Stamatakis, E. \& \& Bull, F. C. (2020). Putting physical activity in the «must-do» list of the global agenda. Br | Sports Med, 54(24). https:/ / doi.org/ 10.1136/ bjsports-2020103509

Thejamovi project. (2021). Jamovi Stats (version1.6) [Computer Softwear] (1.6). https: / / www.jamovi.org

Ulrich, D. A., \& Sanford, C. B. (2000). Test of gross motor development (2nd ed). Pro-ed Austin, TX.

Vleugels, L.W. E., Swinnen, S. P., \& Hardwick, R. M. (2020). Skill acquisition is enhanced by reducing trial-to-trial repetition. Journal of N europhysiology, 123(4), 1460-1471. https:/ / doi.org/ 10.1152/ jn.00741.2019

Wernbom, M., Augustsson, J., \& Thomeé, R. (2007). The influence of frequency, intensity, volume and mode of strength training on whole muscle cross-sectional area in humans. Sports Medicine, 37(3), 225-264. https:/ / doi.org/ 10.2165/ 00007256-200737030-00004

Wing, E. K., Bélanger, M., \& Brunet, J. (2016). Linking parental influences and youth participation in physical activity in- and out-of-school:The mediating role of selfefficacy and enjoyment. American Journal of $H$ ealth Behavior, 40(1), 31-37. https:/ / doi.org/ 10.5993/ AJHB.40.1.4 World Health Organization. (2020). Guidelines on physical activity and sedentary behaviour. https:/ / www. who.int/ publications/ i/ item/ 9789240015128

Zois, J., Bishop, D. J., Ball, K. \& \& Aughey, R. J. (2011). Highintensity warm-ups elicit superior performance to a current soccer warm-up routine. Journal of Science and Medicine in Sport, 14(6), 522-528. https:/ / doi.org/ 10.1016/ j.jsams.2011.03.012 